Publ. RIMS, Kyoto Univ.

15 (1979), 767-772

\title{
Extended Landau Variety and the Singularity Spectrum of Position-Space Feynman Amplitudes
}

By

Takahiro KAWAI

\begin{abstract}
It is shown that the singularity spectrum of (generalized) position-space Feynman amplitude $\Delta_{D}(x ; \lambda)$ is confined to the extended Landau variety (introduced by KashiwaraKawai [8], [9] and Kashiwara-Kawai-Stapp [11] ${ }^{(0)}$ ) associated with the Feynman diagram $D(0)$, which is obtained by replacing all the masses associated with the internal lines of $D$ with zero, at least under the assumption that the diagram $D$ is external.
\end{abstract}

One recent trend in the investigation of analytic properties of the functions important in physics is to investigate the singularity structure of the functions in question from the view point of micro-local analysis, namely, to analyze their singularity structure on the cotangent bundle. (See Pham [15], Sato [16], Iagolnizer [4], [5], [6], Kawai-Stapp [12], [13], K-K-S [11], Sato et al. [18] and references cited there. See also Chandler-Stapp [2] and Iagolnitzer-Stapp [7], whose analysis is essentially of the micro-local character.) In the articles quoted above, the quantity in question is studied in the momentum-space and the celebrated LandauNakanishi variety is naturally the starting point of their analysis. During the coruse of the precise argument on the unitarity integrals and so on, thanks to the harmony between mathematics and physics, K-K-S [11] and Kashiwara-Kawai [8], [9] have been naturally led to the notion of the extended Landau variety which describes the geometric feature of the singularity structure of phase space integrals, the (on-shell) $S$-matrix and Feynman amplitudes (K-K-S [11], Kashiwara-Kawai [8], [9]). Now,

Received September 16, 1977.

* Supported in part by NSF GP 36269. Department of Mathematics, Harvard University and Research Institute for Mathematical Sciences, Kyoto University. Current address: Research Institute for Mathematical Sciences, Kyoto University, Kyoto 606 Japan.

(0) Hereafter referred to K-K-S [11] for short. 
in this note, we observe another interesting property of the extended Landau variety, namely, we show that the singularity spectrum of the generalized position-space Feynman amplitude associated with Feynman diagram $D$ is also described by the extended Landau variety ${ }^{(1)}$ associated with diagram $D(0)$ which is obtained from $D$ by replacing all the masses associated with the internal lines of $D$ with zero. Since the singularity spectrum (=essential support) of a tempered distribution $f(x)$ can be reinterpreted in terms of the growth order conditions on its Fourier transform (Bros-Iagolnitzer [1]), the author hopes that the result in this note will give us an impetus to the further geometric study on the asymptic behavior of the Feynman amplitude in momentum space.

In order to avoid technical difficulties connected with the compactification procedure (see Remark 4 after the proof of the theorem), all the diagrams considered in this article are supposed to be external in the sense that at least one external line is attached to each vertex. Hence we may assume that one and exactly one external line is coming into each vertex. We suppose that all relevant particles are massive and spinless. Note, however, that our final conclusion is not altered even if these conditions are not met. (See Remark 1 after the proof of the theorem.)

In this article, we use the same notations as in Speer [19], Nakanishi [14] and Kawai-Stapp [13] and do not repeat their definitions, if there is no fear of confusions. In the sequel, $\Delta_{l}\left(y ; \lambda_{l}\right)$ stands for the generalized Feynman propagator in the position space (Speer [19]) which is associated with the $l$-th internal line of $D$, that is,

$$
\Delta_{l}\left(y ; \lambda_{l}\right) \equiv \frac{i}{(2 \pi)^{4}} \int \frac{\exp (-i k \cdot y)}{\left(k^{2}-m_{l}^{2}+i 0\right)^{\lambda_{l}}} d^{4} k
$$

Here $y \equiv\left(y_{0}, y_{1}, y_{2}, y_{3}\right)$ is a real four-vector, $k \cdot y=\sum_{\nu=0}^{3} k_{\nu} y_{\nu}, k^{2}=k_{0}^{2}-\sum_{\nu=1}^{3} k_{\nu}^{2}$ and $\lambda_{l}$ is a complex number. The generalized Feynman integral in position space that is associated with $D$ shall be denoted by $A_{D}(x ; \lambda)$, where $\lambda=\left(\lambda_{1}, \cdots, \lambda_{N}\right) \in \mathbb{C}^{N}$. Hereafter we assume that $\lambda_{l}$ is neither zero nor positive integer except Remark 2 below. As usual, $m_{l}$ stands for the mass

(1) Precisely speaking, the extended Landau variety used in this article slightly differs from that introduced in K-K-S [11] and Kashiwara-Kawai [9]. See the definition of $\widetilde{\mathcal{L}}(D(0))$ below for details. 
associated with the $l$-th internal line of $D$. A point in $S^{*} \mathbb{R}^{4 n}$ shall be denoted by $(x ; P)=\left(x_{1}, \cdots, x_{n} ; P_{1}, \cdots, P_{n}\right)$, namely, the $n$-tuple of real four-vectors $P=\left(P_{1}, \cdots, P_{n}\right)$ stands for the cotangent vector at $x=\left(x_{1}, \cdots\right.$, $\left.x_{n}\right) \in \mathbb{R}^{4 n}$.

Theorem. The singularity spectrum ${ }^{(2)}$ S.S. $\Delta_{D}(x ; \lambda)$ is confined to the extended Landau variety $\widetilde{\mathcal{L}}(D(0))$ defined below. Here $D(0)$ denotes a diagram obtained from $D$ by replacing all the internal masses of $D$ with zero and keeping all other structures (such as orientation of the lines etc.) the same as those of $D$.

$\widetilde{\mathcal{L}}(D(0)) \equiv\left\{(x ; \sqrt{-1} P \infty) \in \sqrt{-1} S^{*} \mathbb{R}^{4 n} ;\right.$ there exists a sequence of complex numbers $\alpha_{i}^{(m)}(l=1, \cdots, N)$ and complex four-vectors $x_{j}^{(m)}(j=1$, $\cdots, n)$ and $y_{l}^{(m)}(l=1, \cdots, N)$ which satisfy the following relation (3).\}

$$
\begin{cases}x_{j}^{(m)} \rightarrow x_{j} & (j=1, \cdots, n) \\ \sum_{l=1}^{N}[j: l] y_{l}^{(m)} \rightarrow P_{j} & (j=1, \cdots, n) \\ \sum_{j=1}^{n}[j: l] x_{j}^{(m)}=\alpha_{l}^{(m)} y_{l}^{(m)} & (l=1, \cdots, N) \\ \alpha_{l}^{(m)} y_{l}^{(m) 2} \rightarrow 0 & (l=1, \cdots, N)\end{cases}
$$

Proof. It follows from the definition that the generalized Feynman integral $\Delta_{D}(x ; \lambda)$ in position space has the following form, if $D$ is external.

$$
\Delta_{D}(x ; \lambda)=\prod_{l=1}^{N} \Delta_{l}\left(\sum_{j=1}^{n}[j: l] x_{j} ; \lambda_{l}\right) .
$$

On the other hand, we know (Gel'fand-Shilov [3]) that

$$
\Delta_{l}\left(y ; \lambda_{l}\right)=\frac{\exp \left(-\pi i \lambda_{l}\right) 2^{\lambda_{l}+1} m_{l}^{2+\lambda_{l}}}{(\sqrt{2 \pi})^{4} \Gamma\left(-\lambda_{l}\right)} \frac{K_{2+\lambda_{l}}\left(m_{l}\left(y^{2}+i 0\right)^{1 / 2}\right)}{\left(y^{2}+i 0\right)^{\left(2+\lambda_{l}\right) / 2}}
$$

Here $K_{\nu}(z)$ denotes the modified Bessel function of degree $\nu$. Since $z^{-\nu} J_{\nu}(z)^{(3)}$ is an entire function of $z$, the right hand of (5) is a linear combination of $1,\left(y^{2}+i 0\right)^{1 / 2},\left(y^{2}+i 0\right)^{-\left(2+\lambda_{l}\right)}$ and $\left(y^{2}+i 0\right)^{-\left(3 / 2+\lambda_{l}\right)}$ with analytic coefficients. Therefore we can apply Lemma 1 of Kashiwara-Kawai [8]

(2) See Sato-Kawai-Kashiwara [17] Chapter I for the definition and properties of the singularity spectrum.

(3) $J_{\nu}(z)$ denotes the Bessel function of degree $\nu$. 
to find that the maximum possible set for the singularity spectrum of $\Delta_{D}(x ; \lambda)$ is given by the following set $\widetilde{\mathcal{L}}(D(0))$.

$\widetilde{\mathcal{L}}(D(0))=\left\{(x ; \sqrt{ }-1 P \infty) \in \sqrt{ }-\overline{1} S^{*} \boldsymbol{R}^{4 n} ;\right.$ there exists a sequence of complex numbers $\beta_{l}^{(m)}(l=1, \cdots, N)$ and complex four-vectors $x_{j}^{(m)}(j=1, \cdots$, $n)$ and $Y_{l}^{(m)}(l=1, \cdots, N)$ which satisfy the following relation (6).\}

(6)

$$
\begin{cases}x_{j}^{(m)} \rightarrow x_{j} & (j=1, \cdots, n) \\ \sum_{l=1}^{N}[j: l] \beta_{l}^{(m)} Y_{l}^{(m)} \rightarrow P_{j} & (j=1, \cdots, n) \\ \sum_{j=1}^{n}[j: l] x_{j}^{(m)}=Y_{l}^{(m)} & (l=1, \cdots, N) \\ \beta_{l}^{(m)} Y_{l}^{(m) 2} \rightarrow 0 & (l=1, \cdots, N)\end{cases}
$$

Then, defining $\alpha_{l}^{(m)}$ by $1 / \beta_{l}^{(m)}$ and $y_{l}^{(m)}$ by $\beta_{l}^{(m)} Y_{l}^{(m)}$, we immediately find that (6) can be rewritten in the form of (3). This completes the proof of the theorem.

Remark 1. When $m_{l}=0$, the right hand side of (5) is simplified, namely, it reduces to a constant multiple of $\left(y^{2}+i 0\right)^{-\left(2+\lambda_{2}\right)}$.

Remark 2. If we assume that $\sum_{j=1}^{n}[j: l] x_{j} \neq 0$ for $l=1, \cdots, N$ and that the following equations ( 7$)$ do not admit a non-trivial solution $\beta_{l} \geqq 0$ (i.e. $\beta_{l_{0}} ¥ 0$ for some $\left.l=l_{0}\right)$, then the singularity spectrum of $\Delta_{D}(x ; \lambda)$ can be described by the following analogue (8) of Landau-Nakanishi equations.

$$
\begin{aligned}
& \begin{cases}\sum_{l=1}^{N}[j: l] \beta_{l}\left(\sum_{j^{\prime}=0}^{n}\left[j^{\prime}: l\right] x_{j^{\prime}}\right)=0 & (j=1, \cdots, n) \\
\beta_{l}\left(\sum_{j=1}^{n}\left[j^{\prime}: l\right] x_{j^{\prime}}\right)^{2}=0 & (l=1, \cdots, N)\end{cases} \\
& \begin{cases}P_{j}=\sum_{l=1}^{N}[j: l] \beta_{l} y_{l} & (j=1, \cdots, n) \\
\sum_{j=1}^{n}[j: l] x_{j}=y_{l} & (8 . \mathrm{a}) \\
\beta_{l} y_{l}^{2}=0 & (l=1, \cdots, N) \\
\beta_{l} \geqq 0 & (8 . \mathrm{b}) \\
\beta_{l_{0}} \neq 0 \quad \text { for some } l_{0} & \end{cases}
\end{aligned}
$$

This fact easily follows from Corollary 2.4.2 of Sato et al. [17] Chapter I, $\S 2.4$. That corollary also guarantees that $\Delta_{D}(x ; 1, \cdots, 1)$ is well. 
defined as a hyperfunction in a neighborhood of such a point.

Remark 3. The holonomic character of $\Delta_{D}(x ; \lambda)$, namely, the fact that $\Delta_{D}(x ; \lambda)$ satisfies a holonomic system, can also be verified by making use of Lemma 1 of Kashiwara-Kawai [8]. The characteristic variety of the holonomic system is also described by allowing $(x, P)$ to be complex vectors.

Remark 4. Even when diagram $D$ is not external, we could obtain the same result (only by setting $P_{j_{0}}$ to be zero if the $j_{0}$-th vertex is internal and by requiring (3. a) only for external vertices) if we were to apply Theorem 2.3.1 of Sato et al. [17] Chapter I to $\Delta_{D}$. However, suitable compactification procedure should be needed to make this argument mathematically rigorous. The author hopes to discuss the general case rigorously in the future.

Remark 5. M. Kashiwara and the author have recently found a gap in their original proof of Lemma 2 of their article [8]. Even though they have not yet been able to fulfill the gap, they still conjecture that this should hold, after examining several delicate examples. (KashiwaraKawai $\left[8^{b i s}\right]$ ) If we admit this conjecture, the result of this note is a little improved, that is, we may assume $\alpha_{l}^{(m)}>0$ in (3) besides the conditions given there.

\section{References}

[1] Bros, J. and Iagolnitzer, D., Séminaires Goulaouic-Lions-Schrvartz 1974/1975, exposés 16 and 18.

[2] Chandler, C. and Stapp, H. P., J. Math. Phys. 10 (1969), 826-859.

[3] Gel'fand, I. M. and Shilov, G. E., Generalized Functions, 1. New York and London, Academic Press, 1974. (Translation from the Russian original published in 1958).

[4] Iagolnitzer, D., Analyticity property of scattering amplitudes, a review of some recent developments, Lecture Notes in Physics, 39 (1975), 1-21. Berlin-Heidelberg-New York, Springer.

[5] , Commun. math. Phys. 41 (1975), 39-53.

[6] _-_. Publ. RIMS, Kyoto Univ. 12 supplement (Proc. Oji Seminar) (1977) 89-112.

[7] Iagolnitzer, D. and Stapp, H. P., Commun. math. Phys. 14 (1969), 15-55.

[8] Kashiwara, M. and Kawai, T., Proc. Japan Acad., 52 (1976), 161-164.

[ 8 bis] - Publ. RIMS, Kyoto to Univ, 15 (1979), 551-575. 
[9] , Publ. RIMS, Kyoto Univ. 12 supplement (Proc. Oji Seminar) (1977), 131-140.

[10] - Commum. math. Phys. 54 (1977), 121-134.

[11] Kashiwara, M., Kawai, T. and Stapp, H. P., (referred to as K-K-S[11]), Publ. RIMS, Kyoto Univ. 12 supplement (Proc. Oji Seminar) (1977), 141-146. See also the full paper in Commun, math. Phys. 66 (1979), 95-130.

[12] Kawai, T. and Stapp, H. P., Micro-local study of the S-matrix singularity structure, Lecture Notes in Physics, 39 (1975), 38-48. Berlin-Heidelberg-New York, Springer.

[13] —_ Publ. PIMS, Kyoto Univ. 12 supplement (Proc. Oji Seminar) (1977), 155-232.

[14] Nakanishi, N., Graph Theory and Feynman Integrals. NewYork-London-Paris, Gordon and Breach, 1971.

[15] Pham, F., Microanalyticité de la matrice S., Lecture Notes in Math., 449 (1975), 83-101, Berlin-Heidelberg-New York, Springer.

[16] Sato, M., Recent development in hyperfunction theory and its application to physics., Lecture Notes in Physics, 39 (1975) 13-29, Berlin-Heidelberg-New York, Springer.

[17] Sato, M., Kawai, T. and Kashiwara, M., Microfunction and pseudo-differential equations, Lecture Notes in Math. 287 (1973), 265-529, Berlin-Heidelberg-New York, Springer,

[18] Sato, M., Miwa, T., Oshima T. and Jimbo, M., Publ. RISM, Kyoto Univ. 12 supplement (Proc. Oji Seminar) (1977), 387-438.

[19] Speer, E. R., Generalized Feynman Amplitudes, Princeton, Princeton Univ. Press, 1969.

Added in proof: After this note had been submitted, a related paper titled "Singularities of Feynman diagramans in the coordinate space" by V. A. Smirnov was submitted to Teoreticheskaya i Matematicheskaya Fizika and published in Vol. 36, No. 2, pp. 183-192, Auguat 1978. However, his starting formula (2) is erroneous and it makes his later reasoning ambiguous. His final claim (Theorem in \$10) also contains erroneous statements. 